\title{
Heavy Meson Description with a Screened Potential
}

\author{
P. González ${ }^{(1)}$, A. Valcarce ${ }^{(2)}$, H.Garcilazo $^{(3)}$, and J. Vijande ${ }^{(2)}$ \\ (1) Dpto. de Física Teórica and IFIC \\ Universidad de Valencia-CSIC, E-46100 Burjassot, Valencia, Spain \\ (2) Grupo de Física Nuclear \\ Universidad de Salamanca, E-37008 Salamanca, Spain \\ (3) Escuela Superior de Física y Matemáticas \\ Instituto Politécnico Nacional, Edificio 9, \\ 07738 México D.F., Mexico
}

\begin{abstract}
We perform a quark model calculation of the $b \bar{b}$ and $c \bar{c}$ spectra from a screened funnel potential form suggested by unquenched lattice calculations. A connection between the lattice screening parameter and an effective gluon mass directly derived from QCD is established. Spin-spin energy splittings, leptonic widths and radiative decays are also examined providing a test for the description of the states.
\end{abstract}

Keywords: phenomenological quark models, meson properties

Pacs: 12.39.-x, 14.40.-n, 13.40.Hq 


\section{INTRODUCTION}

Heavy quarkonia, $b \bar{b}$ and to a lesser extent $c \bar{c}$ (for $t \bar{t}$ the weak decay rates are too large for these resonances to be observed), are ideal systems to test quark potential models derived from QCD. Actually for the low-lying states (the ground state for $c \bar{c}$ and the ground and first excited states for $b \bar{b}$ ) one can test the theory itself at the perturbative regime [1]. This may provide a quantitative estimate of the approximations involved when using a potential model interaction derived from QCD to describe heavy quarkonia.

At very short distances a nonrelativistic coulombic potential, with strength proportional to $\alpha_{s}$, the quark-gluon-quark coupling constant, is derived perturbatively from the onegluon exchange interaction in QCD. Relativistic corrections involve spin-independent, spinspin, spin-orbit and tensor terms. Radiative corrections have been estimated as well. At long distances nonperturbative effects involving multigluon interactions, in particular those related to confinement, have to be considered. Lattice calculations in the so-called quenched approximation (only valence quarks) derive a heavy quark long range potential linearly dependent on the interquark distance [2].

The resulting interaction can then be parametrized by a funnel shape coulomb+linear potential. Such a potential has been applied to get a good description of the spectrum of spin-triplet states for $c \bar{c}$ and for the first excited states of $b \bar{b}$ [3]. When completed with some relativistic plus radiative corrections it can give account, through a phenomenological fit of the mass parameters, for the whole meson spectrum ranging from heavy to light mesons, as well as for the electromagnetic, strong and weak decays [4,5].

Further QCD corrections to the coulomb+linear form can be incorporated in the potential through unquenched (valence+sea quarks) lattice calculations [2]. The spontaneous creation of light quark pairs may give rise to a breakup of the color flux tube between the two heavy quarks. It has been proposed that this translates into a screened funnel potential $[2,6,8]$, say the potential does not rise continuously with the interquark distance but it saturates at the splitting energy of the heavy quark pair. Although string breaking can not be said to be definitely confirmed through lattice calculations [7] a quite rapid cross-over from a linear rising to a flat potential is well established in $\mathrm{SU}(2)$ Yang-Mills theories [8]. The analysis of the consequences of such a screening in heavy quarkonia is our main goal in this article. To this purpose we discuss in Sec. II the screened form of the potential and connect it to an effective running gluon mass in QCD. In this form we can obtain from the underlying theory the value of the screening parameter for a given energy scale. Then, via a sensible choice of this scale, we calculate in Secs. III to V the spectra, spin-spin splittings, leptonic widths and radiative decays of $b \bar{b}$ and $c \bar{c}$ mesons. The dependence of our results on the chosen scale is studied in Sec. VI. Finally in Sec. VII we summarize our results.

\section{THE SCREENED FUNNEL POTENTIAL}

The long range behavior of the heavy quark potential can be assigned to the saturation

property of the running QCD coupling constant, $\alpha_{s}\left(Q^{2}\right)$, at the low momentum scale. In a particular framework it has been shown [9], through a solution of the Schwinger-Dyson 
equation, that when decreasing $Q^{2}, \alpha_{s}$ does not increase continuously but instead it saturates at a constant value $\alpha_{s}(0) \simeq 0.8$. Explicitly:

$$
\alpha_{s}\left(Q^{2}\right)=\frac{4 \pi}{\beta_{0} \ln \left[\left(Q^{2}+4 M_{g}^{2}\left(Q^{2}\right)\right) / \Lambda^{2}\right]}
$$

where $\beta_{0}=\left(33-2 n_{f}\right) / 3$, being $n_{f}$ the number of flavors with mass much smaller than $Q$. $\Lambda$ is the QCD scale parameter for which we shall take $300 \mathrm{MeV}$ hereforth and $M_{g}$ is an effective gluon mass given by:

$$
M_{g}^{2}\left(Q^{2}\right)=m_{g}^{2}\left(\frac{\ln \left[\left(Q^{2}+4 m_{g}^{2}\right) / \Lambda^{2}\right]}{\ln \left(4 m_{g}^{2} / \Lambda^{2}\right)}\right)^{-12 / 11}
$$

with $m_{g} \sim 300 \mathrm{MeV}$. Hence the effective gluon mass, $M_{g}$, runs from 0 at $Q^{2} \rightarrow \infty$ to $m_{g}$ at $Q^{2} \rightarrow 0$. Correspondingly $\alpha_{s}$ runs from 0 at $Q^{2} \rightarrow \infty$ (asymptotic freedom) to $\sim 0.8$ at $Q^{2} \rightarrow 0$. By implementing the one-gluon-exchange diagram with $\alpha_{s}\left(Q^{2}\right)$ as given above but substituting $M_{g}^{2}\left(Q^{2}\right)$ by $M_{g}^{2}\left(Q^{2} \rightarrow 0\right) \simeq \Lambda^{2}$, a long range $\left(r>>\Lambda^{-1}\right)$ linear potential comes out [1] (one should keep in mind that fine structure splittings might require a scalar [10] or a mixed scalar-vector structure for the confinement [11]).

This linear tendency gets modified by $q \bar{q}$ pair creation in between the heavy quarks since a screening of the quark color charge at large distances takes place. Moreover the effective coulomb coupling is also affected by the presence of sea quarks. These effects were parametrized in exploratory lattice studies by a screened funnel potential [6]. For the sake of simplicity we shall follow this parametrization though it does not reproduce the rapid turnover around $1 \mathrm{fm}$ from linearly rising to flat potential suggested by modern lattice results [8]. For a meson the potential has the form:

$$
\bar{V}(r)=\left(\bar{\sigma} r-\frac{4}{3} \frac{\bar{\alpha}_{s}}{r}\right)\left(\frac{1-e^{-\mu r}}{\mu r}\right)
$$

where $\mu$ is the screening parameter (with units of an inverse length) and the hat over the parameters in the potential distinguishes them from the nonscreened case: $V(r)=\sigma r-\frac{4}{3} \frac{\alpha_{s}}{r}$. $\bar{V}(r)$ behaves like a coulomb potential for $r \rightarrow 0$ whereas it tends to $\bar{\sigma} / \mu$ for $r \rightarrow \infty$.

Regarding the value of $\mu$ let us realize that for $\mu \rightarrow 0$ one formally recovers the nonscreened potential with the long distance linear behavior one had when doing $M_{g}\left(Q^{2}\right) \sim \Lambda$. On the other hand the confining part of the potential can be written in the form

$$
\bar{V}_{\text {conf }}(r)=\frac{\bar{\sigma}}{\mu}-\bar{\sigma} r \frac{e^{-\mu r}}{\mu r}=\bar{V}_{\text {conf }}(r \rightarrow \infty)-\bar{\sigma} r \frac{e^{-\mu r}}{\mu r}
$$

what suggests that $\mu$ should be connected to an exchanged (Yukawa type) mass. From these considerations we propose this mass to be the effective gluon mass through the identification

$$
\mu=\Lambda-M_{g}
$$

so that for a given scale specified by $Q^{2}$ 


$$
\bar{V}_{\text {conf }}(r)=\frac{\bar{\sigma}}{\mu}-\bar{\sigma} r \frac{e^{-\mu r}}{\mu r}=\frac{\bar{\sigma}}{\mu}-\bar{\sigma} r\left[\frac{e^{-\left(\Lambda-M_{g}\left(Q^{2}\right)\right) r}}{\left(\Lambda-M_{g}\left(Q^{2}\right)\right) r}\right]
$$

This identification establishes a deep connection between the saturation of the coupling constant and the interquark pair creation mechanism both effects governed by $M_{g}\left(Q^{2}\right)$. Therefore $\mu$ runs with $Q^{2}$ so that $0 \simeq \mu\left(Q^{2}=0\right) \leq \mu\left(Q^{2}\right) \leq \mu\left(Q^{2} \rightarrow \infty\right) \simeq 1.52 \mathrm{fm}^{-1}$.

The splitting energy of quark and antiquark in the meson is given by $\bar{\sigma} / \mu$, the maximum value of the potential. No bound state can be found for higher energies due to the breaking of the color flux tube and the most favored subsequent decay in multimeson states. We shall assume that this splitting energy does not depend on flavor or, more precisely, that it does not depend on the scale $Q^{2}$. Hence $\bar{\sigma}$ should also run with the scale $Q^{2}$ in such a way that $\bar{\sigma}\left(Q^{2}\right) / \mu\left(Q^{2}\right) \simeq$ constant. For us $\bar{\sigma}$ will be a free parameter to be determined phenomenologically at a chosen scale. As a value for comparison we could think of the string tension value for the nonscreened case, $\sigma \simeq 1000 \mathrm{MeV} \mathrm{fm}^{-1}$ [12]. A more physical comparison can be done by evaluating, from $\bar{\sigma}, \mu$, and $\bar{\alpha}_{s}$, a hadronic scale $R_{0}$ defined through the force between static quarks at intermediate distances as $F\left(R_{0}\right) R_{0}^{2}=1.65$. For the nonscreened case $R_{0} \simeq 0.5 \mathrm{fm}$ [13]. Concerning the other parameter of the model, $\bar{\alpha}_{s}$, since we have established a link between saturation and screening we shall assume $\bar{\alpha}_{s} \simeq \alpha_{s}$ as given by Eq. (1) at the chosen scale.

Our meson hamiltonian will then be written, up to an additive constant, as:

$$
H=m_{q}+m_{\bar{q}}+\frac{p_{q}^{2}}{2 m_{q}}+\frac{p_{\bar{q}}^{2}}{2 m_{\bar{q}}}+\bar{V}(r)
$$

where the constituent quark and antiquark masses, $m_{q}$ and $m_{\bar{q}}$, are taken as free parameters.

\section{THE $b \bar{b}, c \bar{c}$ AND $c \bar{b}$ SPECTRA.}

In order to apply the screened potential to obtain the $b \bar{b}$ and $c \bar{c}$ spectra we should first identify the $Q^{2}$ scale in each case. For the sake of simplicity and universality let us investigate the possibility to describe $b \bar{b}$ and $c \bar{c}$ mesons with the same set of potential parameter values, say by using the same $Q^{2}$ scale. A sensible guess may be to choose $Q^{2}$ as some intermediate value between the square quark masses $m_{b}^{2}$ and $m_{c}^{2}$. But it turns out that our quark model masses are parameters to be determined from the spectra. Nonetheless we can consider as a reference the central quark mass values ( $\overline{\mathrm{MS}}$ scheme) quoted by the Particle Data Book [14], $m_{b}=4.2 \mathrm{GeV}$ and $m_{c}=1.2 \mathrm{GeV}$, and select $Q^{2}=(2.7 \mathrm{GeV})^{2}$.

Once $Q^{2}$ has been chosen $\alpha_{s}$ and $\mu$ are determined by Eqs. (1),(2) and (5). Then we should establish the protocol to fix the other parameters. We shall fix the quark masses and $\bar{\sigma}$ to get the experimental mass for the ground states of $b \bar{b}$ and $c \bar{c}$ and the first excited state of $b \bar{b}$. Indeed this corresponds to a possible definition of the mass for the confined quarks. Let us note that we do not expect to get the reference $\overline{\mathrm{MS}}$ quark masses since they do not correspond to the same definition but quite different values will not be compatible with the self-consistency of our scheme. The values of the parameters used are compiled in Table I. The resulting spectra, square mean root radii and square quark velocities appear in Tables II, III and IV. 
Let us note that our hamiltonian is spin independent what means that $S=0$ and $S=1$ states are degenerated. Assuming that the energy difference between spin singlet and triplet states comes out mainly due to the spin-spin interaction and taking into account the magnitude of these splittings and the fact that the matrix element for the spin-spin operator $\vec{S}_{i} \cdot \vec{S}_{j}$ is three times bigger for singlets than for triplets, we shall consider our quark model states as close description to spin triplets.

The good agreement of the mass prediction with data for $b \bar{b}$ and $c \bar{c}$ up to $1 \mathrm{GeV}$ excitation energy allows an unambiguous identification of experimental $J^{P}=1^{-}$states: $1 s, 2 s, 1 d, 3 s$ and $4 s$ for $b \bar{b}$, and $1 s$ and $2 s$ for $c \bar{c}$. This is reinforced by the calculation of leptonic width ratios, $\left[\Gamma_{e^{+} e^{-}} / \Gamma_{e^{+} e^{-}}(1 s)\right]$, (see Sec. IV). The same analysis suggests that for $c \bar{c}$ our $1 d$ and $3 s$ states must have some mixing in order to explain the experimental values of the leptonic width ratios, bigger for $1 d$ and smaller for $3 s$ than our approximated results.

For $p$ states and higher energies the situation is much less clear and the interpretation of our results requires some additional considerations. First, relativistic effects, specially for the $c \bar{c}$ system (see the $\left\langle v^{2} / c^{2}\right\rangle$ column in Tables II, III and IV) may be quite relevant: higher order kinetic energy terms increase in importance when increasing the excitation energy, the spin-orbit force can be responsible for the $p$ splittings and for differences between $L=0$ and $L=2$ states, the tensor interaction can induce $s-d$ mixing, the color magnetic potential breaks the degeneracy with $0^{-}$states. Second, coupling to $D$ and $B$ mesons may become relevant [15]. Third, there can be some bias in the results associated to the $Q^{2}$ scale assumed. Anyhow a glance at the experimental leptonic width ratios and their comparison to our calculation, Tables V, suggests that $\Upsilon(10860)$ and $\Upsilon(11020)$ should be mostly $s$ states since for $d$ states the predicted ratios would be three orders of magnitude smaller. For $\psi(4160)$ and $\psi(4415)$ an $s$ state association seems also to be favored, Tables VI and IX.

Concerning $c \bar{b}$ the commented results and the good prediction for the ground state gives us also confidence about the predicted masses of its lower excitations.

The quality of the spectra we get is quite similar to that of other quark model calculations that use strict (nonscreened) confinement [3,4]. The major difference concerns the possible number of quark-antiquark bound states which is finite in our case and infinite for the strict confinement models. This also affects the pattern of energy differences but mostly for higher excited states. Unfortunately the uncertainties associated to the calculation of these states are such that a comparison to data cannot discriminate against any model. The upper energy cut for the spectrum is given in our model by $m_{q}+m_{\bar{q}}+\bar{\sigma} / \mu$. From the values of the parameters we obtain a splitting energy $\bar{\sigma} / \mu \simeq 2070 \mathrm{MeV}$ and the energy thresholds $E_{t h}(b \bar{b}) \simeq 11400 \mathrm{MeV}, E_{t h}(c \bar{b}) \simeq 7973 \mathrm{MeV}$ and $E_{t h}(c \bar{c}) \simeq 4547 \mathrm{MeV}$. Although these values have to be taken very cautiously (actually they are significantly lower than the ones obtained from a Regge analysis [17]) since they depend on the somewhat arbitrary $Q^{2}$ scale (see Sec. VI) the qualitative consequence is clear: the mere existence of a threshold means that a detected state above it would correspond to a wide resonance, a glueball or an exotic state. Moreover, it could even correspond to a narrow resonance provided the existence of selection rules preventing its strong decay.

It is worth to remark that our quark mass values are smaller than the ones obtained from strict confinement models $\left(m_{b} \sim 5000 \mathrm{MeV}, m_{c} \sim 1700 \mathrm{MeV}\right)$ may be indicating that relativistic effects associated to quark pair creation can be taken into account in an effective way, following the philosophy of quark model calculations, via a bigger nonrelativistic quark 
mass. Concerning the value of $\bar{\sigma}\left(1470 \mathrm{MeV} \mathrm{fm}^{-1}\right)$ it is quite big as compared to commonly accepted values for the string tension $\sigma$. However the hadronic scale provided by our model $R_{0} \simeq 0.43 \mathrm{fm}$ is much closer to the nonscreened case. We should also keep in mind that $\bar{\sigma}$ is an effective parameter which may give account of some nonconsidered corrections and that $\bar{\sigma}$ runs with $Q^{2}$ in such a way that the lower the $Q^{2}$ the lower the $\bar{\sigma}$ (see Sec. VI).

\section{A. Singlet-triplet states}

As the screened potential we use has no spin-dependence, spin singlet $(S=0)$ and triplet $(S=1)$ states are degenerated. If we consider the color-magnetic spin-spin term coming from the one-gluon-exchange diagram in QCD and screen it in the same manner than the rest of the potential, i.e.,

$$
V_{s s}=\frac{4}{3} \alpha_{s} \frac{8 \pi}{3 m_{q} m_{\bar{q}}} \vec{S}_{i} \cdot \vec{S}_{j} \delta(\vec{r})\left(\frac{1-e^{-\mu r}}{\mu r}\right)
$$

we realize that when using it in the Schrödinger equation the presence of $\delta(\vec{r})$ gives rise to an unbound low-energy spectrum. This difficulty can be overcome substituting the $\delta(\vec{r})$ by a spreading function depending on a characteristic length. However as this substitution represents also some kind of screening, avoiding the contact interaction, the inverse screening length, $\mu$, appearing in Eq. (8) could not coincide with the value of $\mu$ for the rest of the potential. Then we would be left with two new parameters, the spreading length and a modified inverse screening length, to fit one established and two to be confirmed experimental splitting energies: $M(J / \psi)-M\left[\eta_{c}(1 s)\right], M[\psi(2 s)]-M\left[\eta_{c}(2 s)\right]$ and $M[\Upsilon(1 s)]-M\left[\eta_{b}(1 s)\right]$, what does not seem to be a big deal. Instead we can try to preserve the simplicity of our model and apply first order perturbation theory. Thus the splitting energies obtained from $J / \psi, \psi(2 s)$ and $\Upsilon(1 s)$ are expressed as

$$
\Delta E_{s s}=\frac{4}{3} \alpha_{s} \frac{8 \pi}{3 m_{q} m_{\bar{q}}}|\Psi(0)|^{2}
$$

where $\Psi(0)$ stands for the wave function at the origin for $J / \psi, \psi(2 s)$ and $\Upsilon(1 s)$ respectively. So we predict:

$$
\begin{aligned}
& M(J / \psi)-M\left[\eta_{c}(1 s)\right] \simeq 118.6 \mathrm{MeV} \\
& M[\Upsilon(1 s)]-M\left[\eta_{b}(1 s)\right] \simeq 104.3 \mathrm{MeV} \\
& M[\psi(2 s)]-M\left[\eta_{c}(2 s)\right] \simeq 69.4 \mathrm{MeV}
\end{aligned}
$$

to be compared to the experimental data [14]:

$$
\begin{gathered}
M(J / \psi)-M\left[\eta_{c}(1 s)\right]=117.9 \pm 2.1 \mathrm{MeV} \\
M[\Upsilon(1 s)]-M\left[\eta_{b}(1 s)\right]=160 \pm 40 \mathrm{MeV}
\end{gathered}
$$


and $[18]$

$$
M[\psi(2 s)]-M\left[\eta_{c}(2 s)\right]=64 \pm 12 \mathrm{MeV}
$$

Though we get a remarkable agreement, as the magnitudes of the splittings are not much smaller than the nonperturbed Schrödinger equation eigenvalues we have $[620 \mathrm{MeV}$ for $J / \psi$, $1202 \mathrm{MeV}$ for $\psi(2 s)$ and $131 \mathrm{MeV}$ for $\Upsilon(1 s)]$, specially for $b \bar{b}$, the first order perturbed values should not be taken for granted. Anyhow we can tentatively make some additional predictions for the next two splittings:

$$
\begin{aligned}
& M[\Upsilon(2 s)]-M\left[\eta_{b}(2 s)\right] \simeq 50 \mathrm{MeV} \\
& M[\psi(3 s)]-M\left[\eta_{c}(3 s)\right] \simeq 52 \mathrm{MeV}
\end{aligned}
$$

\section{LEPTONIC WIDTHS}

A complete calculation of $V$ (vector meson) $\rightarrow e^{+} e^{-}$widths involves radiative and relativistic contributions out of the scope of our quark model estimation. Fortunately these corrections can be factorized [19]. Thus for $s$ states we can write the leptonic width $\Gamma_{e^{+} e^{-}}$ as

$$
\Gamma_{e^{+} e^{-}}(n s)=\Gamma_{e^{+} e^{-}}^{(0)}(n s)\left[1-\frac{16 \alpha_{s}}{3 \pi}+\Delta(n s)\right]
$$

The second $\left(-16 \alpha_{s} / 3 \pi\right)$ and third $[(\Delta(n s)]$ terms in the parenthesis on the right hand side stand for the leading order radiative and higher order radiative+relativistic corrections respectively and

$$
\Gamma_{e^{+} e^{-}}^{(0)}(n s)=\frac{16 \pi e_{q}^{2} \alpha^{2}}{M_{n s}^{2}}\left|\Psi_{n s}(0)\right|^{2}
$$

where $e_{q}$ is the electric quark charge, $\alpha$ the fine structure constant, $M_{n s}$ the mass of the $n s$ state and $\Psi_{n s}(0)$ its wave function at the origin.

From Eqs. (9), (18), and (19) we can establish an approximate relation between the correction factors and the quark masses through experimental quantities:

$$
\frac{m_{c}^{2}}{m_{b}^{2}} \frac{\left[1-\frac{16 \alpha_{s}}{3 \pi}+\Delta(1 s)\right]_{c \bar{c}}}{\left[1-\frac{16 \alpha_{s}}{3 \pi}+\Delta(1 s)\right]_{b \bar{b}}} \simeq \frac{\left[\Gamma_{e^{+} e^{-}}(1 s)\right]_{c \bar{c}}}{\left[\Gamma_{e^{+} e^{-}}(1 s)\right]_{b \bar{b}}} \frac{\left[M(\Upsilon(1 s))-M\left(\eta_{b}(1 s)\right)\right]}{\left[M(J / \psi)-M\left(\eta_{c}(1 s)\right)\right]}\left[\frac{M(J / \psi)}{M(\Upsilon(1 s))}\right]^{2}
$$

From Eqs. (18) and (19) it is also clear that a comparison of the calculated $\Gamma_{e^{+} e^{-}}^{(0)}(n s)$ to data will give us the effective magnitude of $\Delta$ needed in our model. So for $b \bar{b}$ we obtain $\Gamma_{e^{+} e^{-}}^{(0)}(1 s)=2.13 \mathrm{keV}$ and $\Delta(1 s)=0.16$ and for $c \bar{c}$ we have $\Gamma_{e^{+} e^{-}}^{(0)}(1 s)=6.37 \mathrm{keV}$ and $\Delta(1 s)=0.36$. We observe that higher order corrections are much more important for the $c \bar{c}$ system as expected. 
As $\Delta$ includes relativistic corrections it depends on the $n s$ state. Inasmuch this dependence is not very strong we could assume a mean value for all these states. Then we could consider the ratio:

$$
\frac{\Gamma_{e^{+} e^{-}}(n s)}{\Gamma_{e^{+} e^{-}}(1 s)} \simeq \frac{\Gamma_{e^{+} e^{-}}^{(0)}(n s)}{\Gamma_{e^{+} e^{-}}^{(0)}(1 s)}=\frac{\left|\Psi_{n s}(0)\right|^{2}}{\left|\Psi_{1 s}(0)\right|^{2}} \frac{M_{1 s}^{2}}{M_{n s}^{2}}
$$

where correction factors cancel. Indeed as far as the masses of the states agree with data this is a test of the ratio of the wave functions at the origin.

Moreover from Eqs. (9) and (21) we can predict

$$
\left[\frac{\Gamma_{e^{+} e^{-}}(2 s)}{\Gamma_{e^{+} e^{-}}(1 s)}\right]_{c \bar{c}} \simeq\left[\frac{M(\psi(2 s))-M\left(\eta_{c}(2 s)\right)}{M(J / \psi)-M\left(\eta_{c}(1 s)\right)}\right]\left[\frac{M(J / \psi)}{M(\psi(2 s))}\right]^{2}
$$

close to the experimental data,

$$
\begin{aligned}
& {\left[\frac{\Gamma_{e^{+} e^{-}}(2 s)}{\Gamma_{e^{+} e^{-}}(1 s)}\right]_{c \bar{c}}=0.41 \pm 0.07} \\
& {\left[\frac{M(\psi(2 s))-M\left(\eta_{c}(2 s)\right)}{M(J / \psi)-M\left(\eta_{c}(1 s)\right)}\right]\left[\frac{M(J / \psi)}{M(\psi(2 s))}\right]^{2}=0.55 \pm 0.04 .}
\end{aligned}
$$

For $d$ states we use:

$$
\Gamma_{e^{+} e^{-}}^{(0)}(n d)=\frac{25 e_{q}^{2} \alpha^{2}}{2 m_{q}^{4} M_{n d}^{2}}\left|R_{n d}^{\prime \prime}(0)\right|^{2}
$$

where $R_{n d}^{\prime \prime}(0)$ stands for the second derivative of the radial wave function at the origin.

Our results, shown in Tables V and VI, are quite encouraging, they seem to confirm the correctness of our assumptions about the validity of the prediction of ratios and support the state identification done in Sec. III (let us comment that there could be some bias due to the $Q^{2}$ scale value chosen, see Tables VIII and IX, Sec. VI).

\section{E1 AND M1 DECAY WIDTHS}

According to the identification of our states with spin triplets done in Sec. III, we shall center in $E 1$ decays in the $b \bar{b}$ and $c \bar{c}$ sectors involving only triplet states, for instance $\chi_{c_{J}}(1 p) \rightarrow \gamma J / \psi, \Upsilon(2 s) \rightarrow \gamma \chi_{b_{J}}(1 p), \ldots$. Then for $i \rightarrow \gamma f,[i(f)$ stands for the initial(final) meson], using a single quark current operator and the nonrelativistic approximation, we can write for the width

$$
\Gamma_{i f}^{E 1}=\frac{4}{27} e_{q}^{2} \alpha k_{i f}^{3}\left(2 J_{f}+1\right) D_{i f}^{2}
$$

where $k_{i f}$ is the photon energy or momentum, $J_{f}$ is the total angular momentum of the final meson and $D_{i f}$ the transition matrix element

$$
D_{i f}=\int_{0}^{\infty} d r u_{i}(r) \frac{3}{k_{i f}}\left[\frac{k_{i f} r}{2} j_{0}\left(\frac{k_{i f} r}{2}\right)-j_{1}\left(\frac{k_{i f} r}{2}\right)\right] u_{f}(r)
$$


being $u_{i, f}(r)$ the reduced radial wave functions for the initial and final mesons respectively and $j_{0}$ and $j_{1}$ spherical Bessel functions. In the limit $k_{i f} r \rightarrow 0$ one recovers the dipole form:

$$
D_{i f} \rightarrow \int_{0}^{\infty} d r u_{i}(r) r u_{f}(r)
$$

In our simple quark model all the $J$ states for $p$ waves, $\chi_{J}$, are degenerated. Let us assume that this degeneration can be dropped out by the consideration of some perturbative interaction such as a spin-orbit one and that first order perturbation theory can give us the right masses. This is quite reasonable since the needed splittings are about one tenth of the energy eigenvalues. Hence we shall use our wave functions (first order perturbation theory does not affect the wave functions) altogether with the experimental masses to get the $E 1$ rates.

Certainly there are relativistic and radiative corrections to the formulas above (involving wave function corrections also). We shall take as a criterium to estimate its importance the value of $k_{i f} R$ ( $R$ can be assimilated to the mean radius of the initial meson) as compared to 1 . Then for the transitions we consider next and from Tables II and III we have $\left(k_{i f} R\right)_{b \bar{b}} \simeq 0.35$ and $\left(k_{i f} R\right)_{c \bar{c}} \geq 1$. Hence we expect a much more reasonable description for $b \bar{b}$ than for $c \bar{c}$. This expectation is confirmed by our results as can be checked in Table VII: the $b \bar{b}$ data are quite well described whereas the $c \bar{c}$ widths deviate by a factor of about 2 .

The much bigger quality of the results we obtain for $b \bar{b}$ as compared to the ones obtained with a simple nonscreened linear potential [3] points out the importance of screening effects. On the other hand by taking into account that with a more refined model without screening [4] both $b \bar{b}$ and $c \bar{c}$ widths are correctly described we can interpret that the effect of screening is taken into account in this model in an effective manner through the fitted parameters.

Less satisfactory from the experimental point of view is the situation for $M 1$ decays since there are only a few experimental $c \bar{c}$ data available $\left[J / \psi \rightarrow \gamma \eta_{c}(1 s)\right.$ and $\left.\psi(2 s) \rightarrow \gamma \eta_{c}(1 s)\right]$. The nonrelativistic theoretical expression for the width is:

$$
\Gamma_{i f}^{M 1}=\frac{16}{3}\left(\frac{e_{q}}{2 m_{q}}\right)^{2} \mu_{q}^{2} \alpha k_{i f}^{3}\left(2 J_{f}+1\right) M_{i f}^{2}
$$

with the transition matrix element $M_{\text {if }}$ given by

$$
M_{i f}=\int_{0}^{\infty} d r u_{i}(r) j_{0}\left(\frac{k_{i f} r}{2}\right) u_{f}(r)
$$

and where we have used an effective magnetic factor $\mu_{q}^{2}$ to take into account corrections to the quark magnetic moment beyond the Dirac particle term.

As the $J / \psi-\eta_{c}(1 s)$ and the $\psi(2 s)-\eta_{c}(2 s)$ mass splittings are reasonably well reproduced by first order perturbation theory we follow the same philosophy as above and assume the same wave function for $J / \psi$ and $\eta_{c}(1 s)$ and analogously for $\psi(2 s)$ and $\eta_{c}(2 s)$. Let us realize that if we fit $\mu_{q}^{2}$ from data this factor can effectively incorporate corrections of the same type mentioned for the $E 1$ case as well as corrections that would come from a better wave function description (specially for singlet states).

Then, for $J / \psi-\eta_{c}(1 s)$, by using that $M_{i f} \simeq 1$ we can estimate $\Gamma_{J / \psi \rightarrow \gamma \eta_{c}(1 s)} \simeq 4.26 \mu_{c}^{2}$. By comparing with the experimental value, $\Gamma_{J / \psi \rightarrow \gamma \eta_{c}(1 s)}=1.2 \pm 0.4 \mathrm{keV}$ we get $\mu_{c} \simeq 0.52$. 
We can compare this value with the corresponding to the second order correction to the magnetic moment operator $[20]\left(1-2 v_{c}^{2} / 3 c^{2}\right) \simeq 0.8$. Since our $\mu_{c}$ has a very effective character the value determined from $\psi(2 s) \rightarrow \gamma \eta_{c}(1 s)$ could be different. If keeping in mind these caveats we assume the same $\mu_{c}^{2}$ we can predict $\Gamma_{\psi(2 s) \rightarrow \gamma \eta_{c}(1 s)} \simeq 1.24 \mathrm{keV}$ to be compared to $\Gamma_{\psi(2 s) \rightarrow \gamma \eta_{c}(1 s)}=0.8 \pm 0.3 \mathrm{keV}$.

Let us also realize that we can get rid of the presence of $\mu_{q}$ by taking the ratio of the widths. Thus we can predict for the non-measured yet $\psi(2 s) \rightarrow \eta_{c}(2 s)$ transition:

$$
\frac{\Gamma_{\psi(2 s) \rightarrow \gamma \eta_{c}(2 s)}}{\Gamma_{J / \psi \rightarrow \gamma} \eta_{c}(1 s)} \simeq\left(\frac{k_{\psi(2 s) \rightarrow \gamma \eta_{c}(2 s)}}{k_{J / \psi \rightarrow \gamma \eta_{c}(1 s)}}\right)^{3} \simeq 0.5
$$

since $M_{i f} \simeq 1$ for both transitions.

Finally for $b \bar{b}$, since we expect relativistic corrections to be much less important, by assuming $\mu_{b} \simeq\left(1-2 v_{b}^{2} / 3 c^{2}\right)=0.94$ (very close to 1 , the value for a Dirac particle), we predict $\Gamma_{\Upsilon(1 s) \rightarrow \gamma \eta_{b}(1 s)}=0.17 \mathrm{keV}$ (we have used $M\left[\eta_{b}(1 s)\right]=9300 \mathrm{MeV}$ [14]; by including the experimental range $M\left[\eta_{b}(1 s)\right]=9300 \pm 20 \pm 20 \mathrm{MeV}$ we have $\Gamma_{\Upsilon(1 s) \rightarrow \gamma \eta_{b}(1 s)}=0.07-0.33$ $\mathrm{keV})$.

\section{THE $Q^{2}$ SCALE}

Although our results are good enough to provide an ad hoc justification for the universal $Q^{2}$ chosen for $b \bar{b}$ and $c \bar{c}$ there can be convenient to try to justify it from more physical grounds. If we write $Q^{2}=-q^{2}$, being $q$ is the momentum transfer between quark and antiquark, we have, in the center of mass system, $Q^{2}=4 \vec{p}_{q}^{2}$ where $\vec{p}_{q}$ is the quark trimomentum $\vec{p}_{q}^{2}=m_{q}^{2} v_{q}^{2} /\left(1-v_{q}^{2}\right)$. By taking as a reference the values of quark masses and velocities previously obtained for $1 s$ states we get $Q_{b \bar{b}}^{2} \simeq(2.9 \mathrm{GeV})^{2}$ and $Q_{c \bar{c}}^{2} \simeq(2.4 \mathrm{GeV})^{2}$, pretty close to the $Q^{2}$ scale value we guessed $Q^{2}=(2.7 \mathrm{GeV})^{2}$. Let us also note that if we had used the nonrelativistic expressions for the trimomentum we would have got $Q_{b \bar{b}}^{2} \simeq(2.8 \mathrm{GeV})^{2}$ and $Q_{c \bar{c}}^{2} \simeq(1.3 \mathrm{GeV})^{2}$ what reflects the much more relativistic character of $c \bar{c}$. Anyhow as we are using a nonrelativistic scheme it can be of help to analyze the variation of our results when a $Q^{2} \simeq(1.3 \mathrm{GeV})^{2}$ scale is chosen for $c \bar{c}$. Actually it is possible to choose $Q_{c \bar{c}}^{2}=m_{c}^{2}=(1357 \mathrm{MeV})^{2}$, so that $\mu=0.556 \mathrm{fm}^{-1}$ and $\bar{\sigma}=1175 \mathrm{MeV} \mathrm{fm}^{-1}$, very close to the string tension value. Furthermore $\bar{\sigma} / \mu=2113 \mathrm{MeV}$, very close to the value obtained with the former universal scale what seems to confirm our assumption about its constancy. The results are shown in Tables VIII and IX. Due to the bigger value of the quark mass the upper energy threshold increases with respect to our former value (4827 $\mathrm{MeV}$ vs 4547 $\mathrm{MeV}$ ). From the comparison with Tables II, III, IV, V and VI, it is clear that the effect of lowering the scale translates in a smaller size and a bigger wave function value (for $n s$ waves) at the origin. The rates $\Gamma_{e^{+} e^{-}}(n s) / \Gamma_{e^{+} e^{-}}(1 s)$ get reduced approaching data. $E 1$ decay rates change also, although by a small amount, in the direction pointed by data. These tendencies though not very significant seem to favor for $c \bar{c}$ a lower scale than the one used in Sec. III. 


\section{SUMMARY}

We have studied heavy quarkonia from a simple screened funnel quark potential model. The form of the potential is suggested by unquenched lattice calculations. The screening inverse length, i. e. the parameter of the potential controlling the onset of screening effects, has been related to an effective gluon mass derived from QCD. As such a mass is also responsible for the saturation property of the running coupling constant in QCD an underlying connection between saturation and screening comes out. The model provides a quite accurate description of $b \bar{b}$ masses, spin-spin splittings, leptonic widths and radiative decays. This may be pointing out the relevance of string breaking effects although the more genuine predictions associated to screening, say the finiteness of the quark-antiquark bound state spectrum, its corresponding upper energy threshold and the breaking color flux tube energy between quarks, remain to be tested in the future since current high energy excitation data do not allow to extract neither quantitative nor qualitative conclusions about their validity.

For $c \bar{c}$ the implementation of other relativistic corrections, apart from screening, seems to be essential to approach data as confirmed by the accurate description provided for more refined models. There could be, in our opinion, interesting to implement screening effects in such kind of models to try to disentangle the role played by relativistic effects of different character.

Let us say finally that the model can be also applied to light hadrons, mesons and baryons, but at the price of introducing more effectiveness in the values of the parameters, losing the stringent connection established with the underlying theory.

\section{ACKNOWLEDGMENTS}

We are specially grateful to Dr. M.A. Sanchis for providing us with phenomenological information. This work has been partially funded by Ministerio de Ciencia y Tecnología under Contract No. BFM2001-3563, by Junta de Castilla y León under Contract No. SA109/01, and by EC-RTN (Network ESOP) under Contract No. HPRN-CT-2000-00130. 


\section{REFERENCES}

[1] F.J. Ynduráin, hep-ph/9910399.

[2] G.S. Bali, Phys. Rep. 343, 1 (2001).

[3] E. Eichten, K. Gottfried, T. Kinoshita, K.D. Lane, and T.M. Yan, Phys. Rev. D 17, 3090 (1978); ibid 21, 203 (1980). E. Eichten and C.Quigg, Phys. Rev. D 52, 1726 (1995).

[4] S. Godfrey and N. Isgur, Phys. Rev. D 32, 189 (1985).

[5] R.K. Bhaduri, L.E. Cohler, and Y. Nogami, Nuovo Cimento A 65, 376 (1981).

[6] K.D. Born, E. Laermann, N. Pirch, T.F. Walsh, and P.M. Zerwas, Phys. Rev. D 40, 1653 (1989).

[7] G.S. Bali et al., Phys. Rev. D 62, 054503 (2000); B. Bolder et al., Phys. Rev. D 63, 074504 (2001); P. Pennanen and C. Michael, hep-lat/0001015; C.W. Bernard et al., Phys. Rev. D 64, 074509 (2001).

[8] P.W. Stephenson, Nucl. Phys. B 550, 427 (1999). F. Knechtli and R. Sommer, Nucl. Phys. B 590, 309 (2000). O. Philipsen and H. Wittig, Phys. Lett. B 451, 146 (1999). P. de Forcrand and O. Philipsen, Phys. Lett. B 475, 280 (2000).

[9] A.C. Aguilar, A. Mihara and A.A. Natale, hep-ph/0109223; J.M. Cornwall and J. Papavassiliou, Phys. Rev. D 40, 3474 (1989); ibid 44, 1285 (1991).

[10] H.J. Snitzer, Phys. Lett. B 76, 461 (1978).

[11] Y.S. Kalashnikova, A.V. Nefediev, Phys. Lett. B 414, 149 (1997).

[12] G. Bali, K. Schilling and A. Wachter, Phys. Rev. D 56, 2566 (1997); hep-ph/9611226.

[13] R. Sommer, Nucl. Phys. B 411, 839 (1994).

[14] K. Hagiwara et al., Phys. Rev. D 66, 010001 (2002).

[15] E. Eichten, Phys. Rev. D 22, 1819 (1980).

[16] S.E. Csorna et al., CLEO collaboration, hep-ex/0207060.

[17] M.M. Brisudová, L. Burakovsky, and T. Goldman, Phys. Rev. D 61, 054013 (2000).

[18] K. Abe et al., Phys. Rev. Lett. 89, 142001 (2002); S.K. Choi et al., Phys. Rev. Lett. 89, 102001 (2002).

[19] W. Buchmuller and S.H.H. Tye, Phys. Rev. D 24, 132 (1981).

[20] T.A. Lähde, C.J. Nyfält, and D.O. Riska, Nucl. Phys. A 645, 645 (1999). 


\section{TABLES}

TABLE I. Parameters of the model.

\begin{tabular}{cc}
\hline \hline $\bar{\alpha}_{s}$ & 0.317 \\
$\mu\left(\mathrm{fm}^{-1}\right)$ & 0.71 \\
$\bar{\sigma}\left(\mathrm{MeV} \mathrm{fm}^{-1}\right)$ & 1470 \\
$m_{b}(\mathrm{MeV})$ & 4664.5 \\
$m_{c}(\mathrm{MeV})$ & 1238.5 \\
\hline \hline
\end{tabular}

TABLE II. $b \bar{b}$ spin-triplet bound state masses (in MeV) and properties. We denote by a '*' the states used to fit the parameters and by a ' $\dagger$ ' experimental states with different possibilities for the orbital angular momentum, it could be $L=0$ and/or $L=2$. Experimental data are taken from Ref. [14] except for that quoted by a ${ }^{* *}$, taken from Ref. [16]. $p$ states are taken as the centroid of the $n p_{0}, n p_{1}$ and $n p_{2}$ experimental data.

\begin{tabular}{lcccc}
\hline \hline & Mass & Exp. & $\left\langle v^{2} / c^{2}\right\rangle$ & $\left\langle r^{2}\right\rangle^{1 / 2}(\mathrm{fm})$ \\
\hline $1 s$ & $9460^{*}$ & $9460.30 \pm 0.26$ & 0.09 & 0.23 \\
$2 s$ & $10023^{*}$ & $10023.26 \pm 0.31$ & 0.08 & 0.51 \\
$1 d$ & 10159 & $10162.2 \pm 1.6^{* *}$ & 0.07 & 0.55 \\
$3 s$ & 10350 & $10355.2 \pm 0.5$ & 0.08 & 0.78 \\
$2 d$ & 10436 & & 0.08 & 0.82 \\
$4 s$ & 10588 & $10580.0 \pm 3.5$ & 0.09 & 1.05 \\
$3 d$ & 10649 & & 0.08 & 1.10 \\
$5 s$ & 10773 & $10865 \pm 8^{\dagger}$ & 0.09 & 1.33 \\
$4 d$ & 10819 & & 0.08 & 1.40 \\
$6 s$ & 10921 & $11019 \pm 8^{\dagger}$ & 0.08 & 1.60 \\
$5 d$ & 10956 & & 0.08 & 1.70 \\
$7 s$ & 11040 & & 0.08 & 1.90 \\
\hline \hline $1 p$ & 9909 & $9900.1 \pm 0.5$ & 0.07 & 0.40 \\
$2 p$ & 10262 & $10260.0 \pm 0.5$ & 0.08 & 0.70 \\
\hline \hline
\end{tabular}


TABLE III. $c \bar{c}$ spin-triplet bound state masses (in MeV) and properties. We denote by a '*, the states used to fit the parameters and by a ' $\dagger$ ' the experimental states with different possibilities for the orbital angular momentum, it could be $L=0$ and/or $L=2$. Experimental data are taken from Ref. [14]. $p$ states are taken as the centroid of the $n p_{0}, n p_{1}$ and $n p_{2}$ experimental data.

\begin{tabular}{lcccc}
\hline \hline & Mass & Exp. & $\left\langle v^{2} / c^{2}\right\rangle$ & $\left\langle r^{2}\right\rangle^{1 / 2}(\mathrm{fm})$ \\
\hline $1 s$ & $3097^{*}$ & $3096.87 \pm 0.04$ & 0.28 & 0.46 \\
$2 s$ & 3679 & $3685.96 \pm 0.09$ & 0.32 & 0.97 \\
$1 d$ & 3794 & $3769.9 \pm 2.5^{\dagger}$ & 0.31 & 1.00 \\
$3 s$ & 4023 & $4040 \pm 10^{\dagger}$ & 0.31 & 1.50 \\
$2 d$ & 4093 & & 0.31 & 1.60 \\
$4 s$ & 4248 & $4159 \pm 20^{\dagger}$ & 0.29 & 2.10 \\
$3 d$ & 4294 & & 0.27 & 2.20 \\
$5 s$ & 4397 & $4415 \pm 6^{\dagger}$ & 0.23 & 2.95 \\
$4 d$ & 4427 & & 0.22 & 3.10 \\
$6 s$ & 4494 & $3525.3 \pm 0.2$ & 0.21 & 3.70 \\
\hline \hline $1 p$ & 3517 & & 0.12 & 0.75 \\
$2 p$ & 3914 & & 0.20 & 1.3 \\
\hline \hline
\end{tabular}

TABLE IV. $b \bar{c}$ spin-triplet bound state masses (in MeV) and properties. Experimental data are taken from Ref. [14].

\begin{tabular}{lcccc}
\hline \hline & Mass & Exp. & $\left\langle v^{2} / c^{2}\right\rangle$ & $\left\langle r^{2}\right\rangle^{1 / 2}(\mathrm{fm})$ \\
\hline $1 s$ & 6362 & $6400 \pm 400$ & 0.18 & 0.37 \\
$2 s$ & 6927 & 0.20 & 0.79 \\
$1 d$ & 7045 & & 0.19 & 0.82 \\
$3 s$ & 7269 & 0.20 & 1.20 \\
$2 d$ & 7342 & 0.20 & 1.25 \\
$4 s$ & 7506 & 0.20 & 1.70 \\
$3 d$ & 7556 & 0.19 & 1.71 \\
$5 s$ & 7677 & 0.17 & 2.16 \\
$4 d$ & 7713 & 0.17 & 2.24 \\
$6 s$ & 7799 & 0.15 & 2.78 \\
$5 d$ & 7825 & 0.15 & 2.89 \\
$7 s$ & 7884 & 0.12 & 3.56 \\
\hline \hline & & & \\
$1 p$ & 6779 & 0.18 & 0.60 \\
& & & \\
$2 p$ & 7163 & 0.20 & 1.02 \\
\hline \hline
\end{tabular}


TABLE V. Leptonic widths $\Gamma_{e^{+} e^{-}}$(in $\mathrm{keV}$ ) for $b \bar{b}$. We denote by a ' $\dagger$ ' the experimental states with different possibilities for the orbital angular momentum, it could be $L=0$ and/or $L=2$. Experimental data are taken from Ref. [14].

\begin{tabular}{lcccc}
\hline \hline & $\Gamma_{e^{+} e^{-}}^{(0)}\left(1-\frac{16 \alpha_{s}}{3 \pi}\right)$ & $\left(\Gamma_{e^{+} e^{-}}\right)_{e x p}$ & $\Gamma_{e^{+} e^{-}}^{(0)} / \Gamma_{e^{+} e^{-}}^{(0)}(1 s)$ & {$\left[\Gamma_{e^{+} e^{-}} / \Gamma_{e^{+} e^{-}}(1 s)\right]_{\text {exp }}$} \\
\hline $1 s$ & 0.98 & $1.32 \pm 0.05$ & 1 & 1 \\
$2 s$ & 0.41 & $0.520 \pm 0.032$ & 0.42 & $0.41 \pm 0.07$ \\
$1 d$ & $3.7 \times 10^{-4}$ & & $3.8 \times 10^{-4}$ & \\
$3 s$ & 0.27 & seen & 0.27 & seen \\
$2 d$ & $5.8 \times 10^{-4}$ & & $5.9 \times 10^{-4}$ & $0.19 \pm 0.03$ \\
$4 s$ & 0.20 & $0.248 \pm 0.031$ & 0.21 & $0.24 \pm 0.06^{\dagger}$ \\
$3 d$ & $6.7 \times 10^{-4}$ & & $6.8 \times 10^{-4}$ & \\
$5 s$ & 0.16 & $0.31 \pm 0.07^{\dagger}$ & 0.16 & $0.10 \pm 0.03^{\dagger}$ \\
$4 d$ & $7.9 \times 10^{-4}$ & & $8.1 \times 10^{-4}$ & \\
$6 s$ & 0.12 & $0.130 \pm 0.030^{\dagger}$ & 0.13 & \\
$5 d$ & $7.1 \times 10^{-4}$ & & $7.3 \times 10^{-4}$ & \\
$7 s$ & 0.10 & & 0.10 & \\
\hline \hline
\end{tabular}

TABLE VI. Leptonic widths $\Gamma_{e^{+} e^{-}}$(in $\left.\mathrm{keV}\right)$ for $c \bar{c}$. We denote by a ' $\dagger$ ' the experimental states with different possibilities for the orbital angular momentum, it could be $L=0$ and/or $L=2$. Experimental data are taken from Ref. [14].

\begin{tabular}{lcccc}
\hline \hline & $\Gamma_{e^{+} e^{-}}^{(0)}\left(1-\frac{16 \alpha_{s}}{3 \pi}\right)$ & $\left(\Gamma_{e^{+} e^{-}}\right)_{\exp }$ & $\Gamma_{e^{+} e^{-}}^{(0)} / \Gamma_{e^{+} e^{-}}^{(0)}(1 s)$ & {$\left[\Gamma_{e^{+} e^{-}} / \Gamma_{e^{+} e^{-}}(1 s)\right]_{\exp }$} \\
\hline $1 s$ & 2.94 & $5.3 \pm 0.4$ & 1 & 1 \\
$2 s$ & 1.22 & $2.19 \pm 0.15$ & 0.42 & $0.41 \pm 0.06$ \\
$1 d$ & 0.026 & $0.26 \pm 0.04^{\dagger}$ & $8.8 \times 10^{-3}$ & $(5.0 \pm 1.1) \times 10^{-2^{\dagger}}$ \\
$3 s$ & 0.76 & $0.75 \pm 0.15^{\dagger}$ & 0.26 & $0.15 \pm 0.04^{\dagger}$ \\
$2 d$ & 0.03 & & $1.1 \times 10^{-2}$ & $0.15 \pm 0.05^{\dagger}$ \\
$4 s$ & 0.43 & $0.77 \pm 0.23^{\dagger}$ & 0.15 & $0.09 \pm 0.03^{\dagger}$ \\
$3 d$ & 0.03 & & $9 \times 10^{-3}$ & \\
$5 s$ & 0.27 & $0.47 \pm 0.10^{\dagger}$ & 0.09 & \\
$4 d$ & 0.02 & & $7.9 \times 10^{-3}$ & \\
\hline \hline
\end{tabular}


TABLE VII. E1 decay widths for $b \bar{b}$ and $c \bar{c}$ (in keV). Experimental data are taken from Ref. [14].

\begin{tabular}{lccccc}
\hline \hline \multicolumn{2}{c}{$b \bar{b}$} & \multicolumn{4}{c}{$c \bar{c}$} \\
\hline Transition & $\Gamma_{E 1}$ & $\Gamma_{\text {exp }}$ & Transition & $\Gamma_{E 1}$ & $\Gamma_{\text {exp }}$ \\
\hline$\Upsilon(2 s) \rightarrow \gamma \chi_{b_{0}}(1 P)$ & 1.46 & $1.7 \pm 0.5$ & $\chi_{c_{0}}(1 P) \rightarrow \gamma J / \psi(1 s)$ & 63.94 & $169 \pm 51$ \\
$\Upsilon(2 s) \rightarrow \gamma \chi_{b_{1}}(1 P)$ & 2.27 & $3.0 \pm 0.8$ & $\chi_{c_{1}}(1 P) \rightarrow \gamma J / \psi(1 s)$ & 125.07 & $295 \pm 71$ \\
$\Upsilon(2 s) \rightarrow \gamma \chi_{b_{2}}(1 P)$ & 2.32 & $3.1 \pm 0.8$ & $\chi_{c_{2}}(1 P) \rightarrow \gamma J / \psi(1 s)$ & 161.64 & $392 \pm 73$ \\
$\Upsilon(3 s) \rightarrow \gamma \chi_{b_{0}}(2 P)$ & 1.71 & $1.4 \pm 0.4$ & $\psi(2 s) \rightarrow \gamma \chi_{c_{0}}(1 P)$ & 62.56 & $26 \pm 5$ \\
$\Upsilon(3 s) \rightarrow \gamma \chi_{b_{1}}(2 P)$ & 2.80 & $3.0 \pm 0.6$ & $\psi(2 s) \rightarrow \gamma \chi_{c_{1}}(1 P)$ & 59.39 & $26 \pm 4$ \\
$\Upsilon(3 s) \rightarrow \gamma \chi_{b_{2}}(2 P)$ & 3.04 & $3.0 \pm 0.6$ & $\psi(2 s) \rightarrow \gamma \chi_{c_{2}}(1 P)$ & 42.39 & $21 \pm 4$ \\
\hline \hline
\end{tabular}

TABLE VIII. $c \bar{c}$ spin-triplet bound state masses (in MeV) and properties from a screened funnel hamiltonian with parameters: $\bar{\alpha}_{s}=0.451, \mu=0.556 \mathrm{fm}^{-1}, \bar{\sigma}=1175 \mathrm{MeV} \mathrm{fm}^{-1}$, and $m_{c}=$ $1357 \mathrm{MeV}$. We denote by a '*' the states used to fit the parameters and by a ' $\dagger$ ' the experimental states with different possibilities for the orbital angular momentum, it could be $L=0$ and/or $L=2$. Experimental data are taken from Ref. [14]. $p$ states are taken as the centroid of the $n p_{0}$, $n p_{1}$ and $n p_{2}$ experimental data.

\begin{tabular}{lcccc}
\hline \hline & Mass & Exp. & $\left\langle v^{2} / c^{2}\right\rangle$ & $\left\langle r^{2}\right\rangle^{1 / 2}(\mathrm{fm})$ \\
\hline $1 s$ & $3097^{*}$ & $3096.87 \pm 0.04$ & 0.28 & 0.42 \\
$2 s$ & $3686^{*}$ & $3685.96 \pm 0.09$ & 0.29 & 0.92 \\
$1 d$ & 3816 & $3769.9 \pm 2.5^{\dagger}$ & 0.07 & 0.97 \\
$3 s$ & 4039 & $4040 \pm 10^{\dagger}$ & 0.30 & 1.41 \\
$2 d$ & 4119 & & 0.15 & 1.47 \\
$4 s$ & 4286 & $4159 \pm 20^{\dagger}$ & 0.30 & 1.92 \\
$3 d$ & 4341 & & 0.18 & 2.00 \\
$5 s$ & 4467 & $4415 \pm 6^{\dagger}$ & 0.28 & 2.50 \\
\hline \hline $1 p$ & 3542 & $3525.3 \pm 0.2$ & 0.11 & 0.72 \\
\hline \hline
\end{tabular}


TABLE IX. Leptonic widths $\Gamma_{e^{+} e^{-}}$(in $\mathrm{keV}$ ) for $c \bar{c}$ with the same model as in Table VIII. We denote by a ' $\dagger$ ' the experimental states with different possibilities for the orbital angular momentum, it could be $L=0$ and/or $L=2$. Experimental data are taken from Ref. [14].

\begin{tabular}{lcccc}
\hline \hline & $\Gamma_{e^{+} e^{-}}^{(0)}\left(1-\frac{16 \alpha_{s}}{3 \pi}\right)$ & $\left(\Gamma_{e^{+} e^{-}}\right)_{e x p}$ & $\Gamma_{e^{+} e^{-}}^{(0)} / \Gamma_{e^{+} e^{-}}^{(0)}(1 s)$ & {$\left[\Gamma_{e^{+} e^{-}} / \Gamma_{e^{+} e^{-}}(1 s)\right]_{\text {exp }}$} \\
\hline $1 s$ & 2.30 & $5.3 \pm 0.4$ & 1 & 1 \\
$2 s$ & 0.87 & $2.19 \pm 0.15$ & 0.38 & $0.41 \pm 0.06$ \\
$1 d$ & $1.3 \times 10^{-2}$ & $0.26 \pm 0.04^{\dagger}$ & $5.5 \times 10^{-3}$ & $(5.0 \pm 1.1) \times 10^{-2^{\dagger}}$ \\
$3 s$ & 0.47 & $0.75 \pm 0.15^{\dagger}$ & 0.20 & $0.15 \pm 0.04^{\dagger}$ \\
$2 d$ & $1.72 \times 10^{-2}$ & & $7.4 \times 10^{-3}$ & \\
$4 s$ & 0.34 & $0.77 \pm 0.23^{\dagger}$ & 0.15 & $0.15 \pm 0.05^{\dagger}$ \\
$3 d$ & $1.74 \times 10^{-2}$ & & $8.2 \times 10^{-3}$ & \\
$5 s$ & 0.24 & $0.47 \pm 0.10^{\dagger}$ & 0.10 & $0.09 \pm 0.03^{\dagger}$ \\
\hline \hline
\end{tabular}

